\title{
On Problems in SPOC Teaching and Solution Strategy-With 'Introduction to Chinese Traditional Culture' in University of Science and Technology as An Example
}

\author{
Kaiyue Bian \\ PLA University of Science and Technology College of Science, Nanjing 21000,China, \\ biankaiyue_bky@163.com
}

Keywords: Mlitary education, Online teaching, POC teaching.

\begin{abstract}
SPOC belongs to a hybrid teaching mode integrating online education and offline entity classroom teaching. It is also an important measure of university of science and technology to promote education teaching reform. 'Introduction to Chinese Traditional Culture' is listed as online teaching demonstration course. Some teachers' teaching concept has not been completely transformed as a new teaching mode pilot. The positioning is not clear. Contradictions and many problems in SPOC promotion process are deeply analyzed. Teaching practice of 'Introduction to Chinese Traditional Culture' course is combined to propose many solution strategies including change of teaching idea, transformation of role positioning, quantity enriching, classification according to materials, perfection of function module, etc.
\end{abstract}

\section{Introduction}

A hybrid teaching mode integrating online education and offline entity classroom teaching —SPOC (Small Private Online Course) may become a more popular teaching mode with gradual subsiding of MOOC mania. In June 2014, SPOC teaching pilot is implemented in university of science and technology. In 2015, 'Introduction to Chinese Traditional Culture' and other course are listed as online teaching demonstration course. The author regards the course as an example for discussing problems in SPOC teaching practice and solution strategy.

\section{SPOC and its development situation}

\subsection{Connotation of SPOC}

SPOC refers to 'small-scale restrictive online course'. [1] In 2013, professor Fox-head of MOOC in University of California Berkeley Branch (Armando Fox) proposed the SPOC concept for the first time. He firstly implemented SPOC teaching experiment in University of California Berkeley Branch. Its basic flow is shown as follows: teachers distributed video and other digital resources to students as homework. Then, knowledge which has been mastered and not mastered by students was comprehended in classroom teaching. Questions of students were answered on the class. Homework and other tasks were handled with students together. Excellent online course resources were organically combined with classroom teaching, thereby transforming teaching process, and improving teaching efficiency.

\subsection{Advantages of SPOC}

SPOC has obvious advantages compared with traditional classroom teaching. Teachers should prepare the most basic and core elements in teaching contents as modularity and topical digital resources, which are published to the platform. Students can autonomously learn teaching core contents in advance; teachers can spend more time and energy to in-depth explanation or discussion on the classroom. Classroom teaching center of gravity is changed from shallow knowledge transfer with teachers as main body to information absorption, internalization, thinking and creation with students as main body. Teachers lead students to more targeted and in-depth discussion, thereby cultivating their comprehensive ability to solve problems, express views, etc. 
MOOC teaching content, teaching form, education concept and technology platform are improved by SPOC compared with MOOC, SPOC also has obvious advantages. MOOC course is suitable for different learning groups. SPOC updates classroom teaching mode. It not only makes teachers return to class and become real master, but also gives students complete and in-depth learning experience, thereby enhancing students' learning motivation, and being conducive to improve the course completion efficiency. SPOC student scale is smaller; thereby teachers and students can exchange and reply one to one. The problem of MOOC peer mutual assessment can be solved fundamentally, namely 'teaching' is available rather than 'education'. In addition, privacy features can may students qualified in application to produce sense of pride, sense of responsibility and urgency of possession of scarce resources so as to improve their degree of importance to learning, and enhancing learning motivation.

\subsection{Present situation of SPOC development}

Professor Fox provided 'software engineering' course to students in Berkeley Campus in 2013 in the mode of SPOC. Students submitted complete programming assignments or configured complete application procedure in the cloud, who can immediately obtain detailed scoring results and more detailed feedback. Fox team moved the SPOC mode to Binghamton University, University of Hawaii, University of Colorado and University of North Carolina in the spring of 2013 because of remarkable achievements. Harvard University also implemented SPOC teaching in law college, Kennedy School and design institute. California SAN Jose State University in Silicon Valley and Bangkeshan Community College in Boston respectively utilized courses of Massachusetts Institute of Technology on edX platform for SPOC teaching experiment, and remarkable results were achieved. Tsinghua University studied and developed since 2011, which firstly created SPOC platform - 'Zhixueyuan'. 'College Physics' and other courses are launched. Complete teaching resources are configured. The first batch of courses was formally online in September 2013. Application of SPOC mode in China was started. [2 The first batch of trial schools of Zhixueyuan includes dozens of colleges and universities such as Tsinghua University, China University of Geosciences, Southwest Jiaotong University, etc. The platform is based on teaching material as the origin, which is supported by knowledge system. It has unique features in the aspects of resources organization mode, data analysis model, teaching management module, content representation form, learning process support, etc. Polytechnic University implemented SPOC online teaching pilot firstly in June 2014. Nearly 40 courses were listed into SPOC demonstration course construction in 2015.

\section{Problems in 'Introduction to Chinese Traditional Culture' SPOC teaching}

\subsection{Teaching concept is not completely changed, and the online teaching 'form' is more than content.}

The course 'Introduction to Chinese Traditional Culture' is adopted as an example. Some teachers can not actually apply online teaching concept and modern education technology real teaching in the process of online teaching. Classroom teaching content and teaching contents produced on the platform are mutual different, some teachers simply believe that the teaching quality can be improved greatly if a lot of data is uploaded to the platform, the interaction between teachers and students is converted into network interaction question. Modern education technology is introduced in teaching rather than simple replacement of teaching means, concepts, mode and methods should be linked for change.

3.2 Teacher role positioning is limited, and effective design of student learning activity is deficient.

Current online teaching is only limited to online answering, which is lack of scheduled organization and guidance of teachers on discussion activities as well as attention on learning activity of students, thereby group learning and division cooperation are only limited in form. SPOC online teaching should include the follows: teaching objective design and realization process and ability obtained by students in the process.

\subsection{Autonomous learning resources are not rich with weak pertinence.}


At present, SPOC online teaching is only related to whether students can learn by themselves or not in the aspect of autonomous learning, more learning resources are not provided for them, and it only focuses on how of students learn by themselves. It is mainly embodied in two aspects: though course group has uploaded a lot of data of each subject of the course, the required data depth and width are not enough if students want to deeply study some question due to limitation of objective condition. Related specific data are accumulated on the platform, the extension data are not classified according to students' recognition level and knowledge reserve due to limitation of teachers' own level in the course group. Therefore, students have no idea in face of a lot of extension data.

\subsection{The platform function module cannot be suitable for diversified teaching requirements}

Although SPOC platform module function is quite powerful, it is able to complete basic requirement of online teaching, there are still two problems from long-term perspective. Firstly, forum capacity is too small. For example, only video smaller than $5 \mathrm{M}$ is allowed for uploading, thereby most videos produced by students can not be uploaded, thereby homework mutual assessment is affected, and students' learning enthusiasm and initiative are affected. Secondly, question module form is traditional with no feedback function. Currently, online test topic form is generally objective topic (blank filling, selection and true-false) and subjective topic (short answer and discussion). Solution practice based on case and question is not sufficient. It still focuses on checking students' mastering degree on knowledge. It is far different from the direction requirements of humanistic courses. In addition, the platform just provides answering results without proper analysis and comment, and it is also not provided with feedback functions (including teacher feedback and student feedback), thereby greatly limiting students' creative thinking and reorganization ability.

\section{Strategy of 'Introduction to Chinese Traditional Culture' to improve SPOC teaching efficiency}

\subsection{Change of teaching idea}

After network technology was introduced into teaching, the method of teaching and learning in the classroom are changed. Traditional education mode with teacher as center, classroom as center and examination response as target will be replaced by modern education mode with practice as center and the objective of obtaining lifelong learning and constantly updating knowledge ability. Everyone must learn at any time, and constantly update. Teachers should firstly update teaching ideas; they can adapt to requirements of modern information technology and course integration only by constantly learning the advanced education idea, teaching design theory and method. Online education does not refer to networking of traditional education. It is not a teaching method but a complete system. The system is composed of three parts of teaching resource acquisition, utilization and development, assessment and identification of student knowledge formation and skills, quality control and guarantee of teacher-student interaction. Teachers should select suitable learning contents suitable for teaching in massive information and data, the contents uploaded in the platform is organically combined with classroom teaching. Teaching information transfer is organically combined with learning absorption, thereby actually implementing online teaching, and exerting the role of online teaching.

\subsection{Conversion role positioning}

Firstly, teachers become guider and designer of students' learning activity. Modern teaching theory believes that education has essential attribute of value guidance by teachers and dialectical unity of student independent construction. Teachers guide students to design appropriate learning activities, and help them to further explore required prior experience so as to realize high level application of curriculum resource value and help them to actually understand and master basic knowledge and basic skills in the process of independent exploration and cooperation exchange. The course group of 'Introduction to Chinese Traditional Culture' designs learning frame and direction of each topic for students through guidance plan before, during and after class. Teachers should guide students for independent exploration and exchange learning from the perspectives of thinking and 
method mainly, thereby achieving inclusion rather than exposure, indication rather than clear description, openness rather than fullness, and guidance rather than distribution. Teacher role is changed from 'actor' in traditional classroom teaching into 'director' of the whole teaching activities.

Secondly, teachers also become organizers and partners of students' learning activities. Organization refers that teachers create autonomous, inquiry and cooperation space for students, and students are organized to discover, seek, search and utilize learning resources under the context of modern education theory. Students are organized for creating and keeping active mental atmosphere, etc. in the classroom and learning process. Online learning progress and difficulties should be selected by students according to own practical condition. It is required that students should be conscious, and teachers need to penetrate through the learning process of students. They should assist students to overcome inertia through activity organization guidance and incentives, thereby forming sound learning system and learning habits suitable for students themselves and students can be constantly motivated for continuous development at different learning stages.

\subsection{Quantity enrichment and classification based on materials}

The data quantity and quality uploaded on the platform are not enough from the perspective of course 'Introduction to Chinese Traditional Culture'. Course group should strengthen in-depth development of existing resources; the existing materials should be concretely divided and classified according to easiness, medium level and difficulty of students' cognitive level. Most existing resources are directly introduced with weak pertinence. The resources should be systematically process and further processed according to practical condition of students aiming at teaching objectives. Or a series of video and radio database and question bank with independent knowledge property are created based on students' cognitive level. Development of autonomous learning resources based on the course is strengthened, the width and depth of autonomous learning resource development should be improved, and the breadth quantity of resources determines the resource application face. While the depth and precision of resource determines the application level and final application effect thereof.

\subsection{Improvement of module function}

It is urgent to improve two aspects based on actual situation of current platform application: firstly, memory capacity of teaching forum is expanded, or similar virtual network backup is established, large individual video can be uploaded and saved on the forum conveniently; secondly, feedback function module is increased, after each function module is clicked, the user can enter the bottom end of the page from the perspective of system structure design, the module of 'my suggestion' is increased as a window for perfecting soliciting suggestion of each module, 'interaction module' is increased on the testing question page from the perspective of internal structure design. Therefore after the answer of the students is opened, students and teaches can exchange and interact aiming at the answer, thereby improving learning interests and efficiency.

Online learning is limited by interaction, traditional learning is limited by time and space, and SPOC teaching realized perfect combination of online learning and traditional learning. Environment and condition, resource and contents, method and strategy are provided for students to transit from shallow learning to in-depth learning. [3] SPOC online teaching implementation condition in recent two years shows that online teaching is extension of classroom teaching, online teaching and classroom teaching are mutually penetrated to form consensus. SPOC online teaching is more and more focused as important means and tool of teaching reform in university of science and technology. Transformation of teaching idea, breadth and depth development of autonomous learning resources and online teaching evaluation feedback system establishment have become important factors of online teaching. Treatment of the aspects has very important significance to further promote development of online teaching. 


\section{References}

[1] Zhu Zhiting. New pattern of online learning at 'Houmuke' period [N]. China Education News, 2014-05-21 (11).

[2] Zheng Qi. SPOC: Integration and innovation of combing with college teaching [J]. Physics and Engineering, 2014 (1): 15-18.

[3] Zeng Mingxing, Li Guiping. From MOOC to SPOC: Construction of a deep learning mode [J]. Theory and dispute 2015 (11) : 28 and 35. 